\title{
INERTIA EFFECTS IN A MULTILOBE CONICAL BEARING LUBRICATED WITH A COUPLE STRESS FLUID
}

\author{
A. WALICKA*, E. WALICKI and P. JURCZAK \\ University of Zielona Góra, Faculty of Mechanical Engineering \\ ul. Szafrana 4, 65-516 Zielona Góra, POLAND \\ E-mail: A.Walicka@ijame.uz.zgora.pl
}

\begin{abstract}
In this paper, a multilobe conical bearing is analyzed. A lubricant modelled by a couple stress fluid flows in the bearing clearance. The Galerkin method is used to determine the mechanical parameters of multilobe journal bearings. An example of a two-lobe conical bearing is discussed in detail. The inertia of the flowing lubricant is taken into account in the analysis. It has been found that the increase of the couple stress generates an increase the pressure in the clearance.
\end{abstract}

Key words: inertia effects, modified Reynolds equation, Galerkin method, mechanical parameters of bearing, additives.

\section{Introduction}

Many researchers have investigated the steady laminar flows of a Newtonian fluid in a clearance of a bearing with nonporous surfaces. The bearing walls were modelled as two conical or spherical surfaces, two disks and two cylindrical surfaces (for journal bearings). In a general case, the bearings are modeled by two surfaces of revolution [1-4].

With the development of modern machine elements the use of complex fluids as lubricants has become of great interest. They also reduce the coefficient of friction and increase the temperature range in which the bearing can operate.

The development of physico-chemistry has led to the introduction of new lubricants which enhance the conditions of the bearing operation and its performance. The mono and multigrade lubricants used in present-day tribological applications are blended with additives which are polymeric in nature. This blending results in a deviation of lubricant rheology from Newtonian to non-Newtonian.

The study of multi-component oils has included such models as: micropolar fluids [5], couple-stress fluids [2-4, 6], viscoplastic fluids [7, 8] and pseudoplastic fluids [9-12].

A more general list of the rheological models of modern lubricants can be found in the works [3, 4].

In recent years, the increase in the ratio of the transmitted power to the mass of rotating mechanisms has led to a reduction of the bearings size and increased rotational speed of the bearings. It is convenient to use conical bearings to increase the mechanical performance of the machines. In this group, multilobe conical bearings stand out for their stable dynamic characteristics [13-18].

In this paper, the Galerkin method was applied to solve the Reynolds equation for the multilobe conical bearing. In this analysis, the inertia effects of the flowing lubricant in the bearing clearance were considered. The lubricant was modelled as a couple-stress fluid.

\footnotetext{
* To whom correspondence should be addressed
} 


\section{Analysis of a lubricant flow in a bearing clearance} function:

Figure 1 shows the bearing configuration. The surface of the bearing shaft is described by the

$$
R(\hat{x})=\hat{x} \sin \alpha,
$$

which denotes its radius. Let the shaft spin at angular speed $\omega$; the bearing thickness is described by the function $h=h(\hat{x}, \vartheta)$. Let $M$ denotes the geometrical parameter of the thickness defined by the relation

$$
M=\frac{h_{M}}{h_{m}}
$$

where $h_{m}$ is the minimum thickness of the bearing clearance, but $h_{M}$ is the maximum thickness of the bearing clearance.

Denoting by $\psi$ the arc span of the lobe one can express the bearing clearance in the following form

$$
h=h_{M} H(M, \psi, \vartheta)
$$

Note that there are the following relations:

- for $\vartheta=0$

$$
H(M, \psi, 0)=1 \quad \text { and } \quad h=h_{M},
$$

- for $\vartheta=\psi$

$$
H(M, \psi, \psi)=\frac{1}{M} \quad \text { and } \quad h=h_{m}=\frac{h_{m}}{M} .
$$

a)

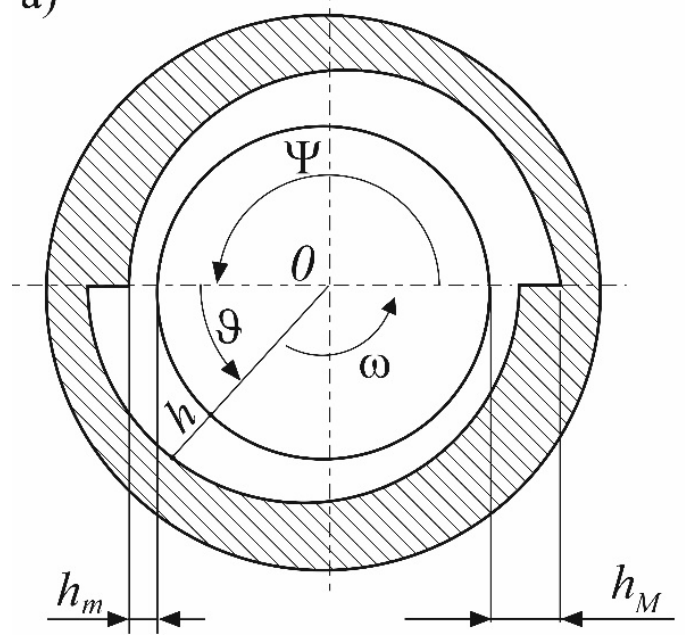

b)

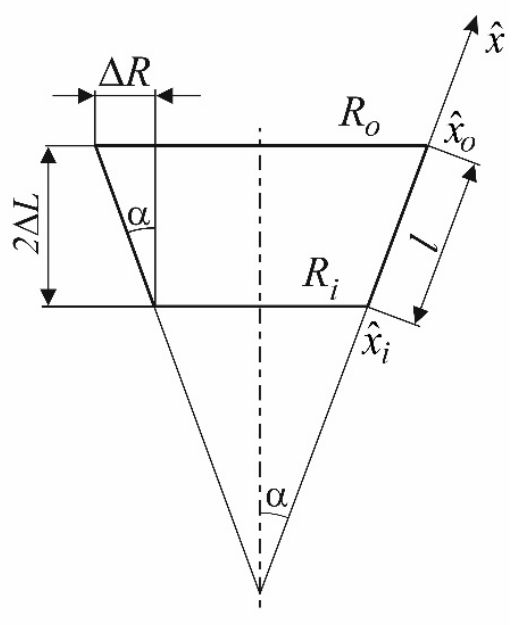

Fig.1. Geometry of a multilobe conical bearing. 
For a multilobe conical bearing we can express the thickness of the bearing clearance in the following form

$$
h=h_{M} M=h_{M}\left(1-\frac{M-1}{M} \frac{\vartheta}{\psi}\right)
$$

where

$$
H=1-\frac{M-1}{M} \frac{\vartheta}{\psi}, \quad \psi=\frac{2 \pi}{N}
$$

where $N$ denotes the number of the bearing lobes.

The Reynolds equation - for the curvilinear thrust bearing - with inertia effects for the lubricant modelled by a couple stress fluid has the form [1-4]

$$
\begin{aligned}
& \frac{1}{R} \frac{\partial}{\partial \hat{x}}\left[R h^{3} f(l, h) \frac{\partial p}{\partial \hat{x}}\right]-\frac{3}{10} \rho \omega^{2} \frac{1}{R} \frac{\partial}{\partial \hat{x}}\left[R^{2} R^{\prime} h^{3} g(l, h)\right]+ \\
& +\frac{1}{R^{2}}\left[h^{3} f(l, h) \frac{\partial p}{\partial \vartheta}\right]=6 \mu \omega \frac{\partial h}{\partial \vartheta}
\end{aligned}
$$

where

$$
\begin{aligned}
& f(l, h)=1-12\left(\frac{l}{h}\right)^{2}+24\left(\frac{l}{h}\right)^{3} \operatorname{th}\left(\frac{h}{2 l}\right), \\
& g(l, h)=1+40\left(\frac{l}{h}\right)^{3}\left[\operatorname{th}\left(\frac{h}{2 l}\right)-\frac{h}{3 l}\right],
\end{aligned}
$$

and

$$
l=\left(\frac{\eta}{\mu}\right)^{\frac{1}{2}}, \quad R^{\prime}=\frac{d R}{d \hat{x}}
$$

here $\mu$ is the shear viscosity (otherwise called Newtonian dynamic viscosity), $\eta$ is the additional viscosity associated with the presence of the couple stress.

The practical values of the relation $\frac{l}{h}$ are contained in the interval

$$
0 \leq \frac{l}{h} \leq 0.2
$$

This assumption allows us to assume approximate values for the function $f(l, h)$ and $g(l, h)$

$$
f(l, h) \approx 1-12\left(\frac{l}{h}\right)^{2} ; \quad g(l, h) \approx 1-\frac{40}{3}\left(\frac{l}{h}\right)^{2}
$$

taking into account relations (2.2), (2.5) and (2.7) we may write 


$$
f(l, h) \approx 1-12 \frac{l^{* 2}}{M^{2} H^{2}}, \quad g(l, h) \approx 1-\frac{40}{3} \frac{l^{* 2}}{M^{2} H^{2}}, \quad l^{*}=\frac{l}{h_{m}} .
$$

Introducing the following notations

$$
\begin{array}{ll}
\varepsilon=\frac{R_{i}}{R_{o}}, \quad R_{a}=\frac{R_{o}+R_{i}}{2}=\frac{R_{o}}{2}(1+\varepsilon), & \zeta=\frac{R_{o}+R_{i}}{2 R_{a}}=\frac{1-\varepsilon}{1+\varepsilon}, \\
\lambda=\frac{\Delta L}{R_{a}}, \quad \Lambda=\frac{6 \mu \omega R_{a}^{2}}{h_{M}^{2}}, \quad P=\frac{p}{\Lambda}, & R=R_{a}(\zeta x+1), \\
x=\frac{2 R}{R_{o}-R_{i}}-\frac{R_{o}+R_{i}}{R_{o}-R_{i}}=\frac{1}{\zeta}\left(\frac{R}{R_{a}}-1\right), & I=\frac{1}{20} \frac{\rho \omega^{2} h_{M}^{2}}{\mu}, \\
\sin ^{2} \alpha=\frac{\zeta^{2}}{\zeta^{2}+\lambda^{2}}, \quad \frac{\partial}{\partial \hat{x}}=\frac{\sin \alpha}{R_{a} \zeta} \frac{\partial}{\partial x}, & \beta=\frac{l^{*}}{M}
\end{array}
$$

and taking into account relations (2.12) we may express Eq.(2.8) in the following form

$$
\begin{aligned}
& \frac{\zeta x+1}{\zeta^{2}+\lambda^{2}} \frac{\partial}{\partial x}\left[(\zeta x+1)\left(H^{3}-12 \beta^{2} H\right) \frac{\partial P}{\partial x}\right]-\frac{I \zeta}{\zeta^{2}+\lambda^{2}}(\zeta x+1) \frac{\partial}{\partial x}\left[( \zeta x + 1 ) ^ { 2 } \left(H^{3}+\right.\right. \\
& \left.\left.-\frac{40}{3} \beta^{2} H\right)\right]+\frac{\partial}{\partial \vartheta}\left[\left(H^{3}-12 \beta^{2} H\right) \frac{\partial P}{\partial \vartheta}\right]=(\zeta x+1)^{2} \frac{\partial H}{\partial \vartheta}
\end{aligned}
$$

where $I$ is the inertia parameter, $x$ is a new variable contained in the interval $<-1,+1\rangle$.

\section{Solution to the Reynolds equation}

A solution to Eq.(2.14) will be searched - using the Galerkin method - in the form of a product [3, 4, 18]

$$
P=P_{\infty}(\vartheta) f(x)
$$

where the function $f(x)$ takes the following form

$$
f(x)=\sum_{m=1}^{n} C_{m} f_{m}(x)
$$

but $P_{\infty}(\vartheta)$ is the solution to the simplified form of Eq.(2.14)

$$
\frac{\partial}{\partial \vartheta}\left[\left(H^{3}-12 \beta^{2} H\right) \frac{\partial P_{\infty}}{\partial \vartheta}\right]=\frac{\partial H}{\partial \vartheta}
$$

describing the pressure distribution in the cylindrical bearing. Integrating Eq.(3.3) we may obtain 


$$
P_{\infty}(\vartheta)=\int_{0}^{\vartheta} \frac{\left(H-H^{*}\right)}{\left(H^{3}-12 \beta^{2} H\right)} d \vartheta
$$

where $H^{*}$ is the lubricant film thickness when the pressure $P_{\infty}(\vartheta)$ reaches the maximum values.

To determine $H^{*}$ we assume that $P_{\infty}(\psi)=0$ [3]; then

$$
H^{*}=\frac{\int_{0}^{\psi} \frac{H d \vartheta}{\left(H^{3}-12 \beta^{2} H\right)}}{\int_{0}^{\psi} \frac{d \vartheta}{\left(H^{3}-12 \beta^{2} H\right)}} .
$$

Taking into account relationships (3.1) and (3.2) we will search for the solution to Eq.(2.14) in the following form $[3,4,18]$

$$
P=P_{\infty}(\vartheta) \sum_{m=1}^{n} C_{m} f_{m}(x)
$$

which must satisfy the following equation

$$
\begin{aligned}
& \int_{0}^{\psi+1} \int_{-1}^{+1}\left\{\frac{\zeta x+1}{\zeta^{2}+\lambda^{2}} \frac{\partial}{\partial x}\left[(\zeta x+1)\left(H^{3}-12 \beta^{2} H\right) \frac{\partial P}{\partial x}\right]+\right. \\
& -\frac{I \zeta}{\zeta^{2}+\lambda^{2}}(\zeta x+1) \frac{\partial}{\partial x}\left[(\zeta x+1)^{2}\left(H^{3}-\frac{40}{3} \beta^{2} H\right)\right]+ \\
& \left.+\frac{\partial}{\partial \vartheta}\left[\left(H^{3}-12 \beta^{2} H\right) \frac{\partial P}{\partial \vartheta}\right]\right\} P_{\infty}(\vartheta) f_{i}(x) d \vartheta d x=\int_{0}^{\psi+1} \int_{-1}^{+1}\left[(\zeta x+1)^{2} \frac{\partial H}{\partial \vartheta}\right] P_{\infty}(\vartheta) f_{i}(x) d \vartheta d x
\end{aligned}
$$

Calculating the integrals in Eq.(3.7) we get

$$
\sum_{m=1}^{n} C_{m} A_{m i}+G_{\vartheta}\left(\frac{\zeta}{\zeta^{2}+\lambda^{2}} \sum_{m=1}^{n} C_{m} B_{m i}+\frac{1}{\zeta^{2}+\lambda^{2}} \sum_{m=1}^{n} C_{m} D_{m i}\right)=\left(1+\frac{2 I \zeta^{2}}{\zeta^{2}+\lambda^{2}} G_{I}\right) F_{i}
$$

where

$$
\begin{aligned}
& A_{m i}=\int_{-1}^{+1} f_{m}(x) f_{i}(x) d x, \quad B_{m i}=\int_{-1}^{+1}(\zeta x+1) \frac{d f_{m}(x)}{d x} f_{i}(x) d x, \\
& D_{m i}=\int_{-1}^{+1}(\zeta x+1)^{2} \frac{d^{2} f_{m}(x)}{d x^{2}} f_{i}(x) d x, \quad F_{i}=\int_{-1}^{+1}(\zeta x+1)^{2} f_{i}(x) d x,
\end{aligned}
$$


but the coefficients $G_{\vartheta}$ and $G_{I}$ are given by the relationships

$$
\begin{aligned}
G_{\vartheta}= & \frac{\int_{0}^{\psi}\left(H^{3}-12 \beta^{2} H\right)\left[P_{\infty}(\vartheta)\right]^{2} d \vartheta}{\int_{0}^{\psi} \frac{\partial H}{\partial \vartheta} P_{\infty}(\vartheta) d \vartheta}, \\
G_{I}= & \frac{\int_{0}^{\psi}\left(H^{3}-\frac{40}{3} \beta^{2} H\right) P_{\infty}(\vartheta) d \vartheta}{\int_{0}^{\psi} \frac{\partial H}{\partial \vartheta} P_{\infty}(\vartheta) d \vartheta} .
\end{aligned}
$$

The unknown coefficients $C_{m}$ depend on the form of functions $f_{k}(x)$ approximating the pressure distribution along the conical bearing generatrix; when we assume the trigonometric form for $f_{k}(x)$

$$
f_{k}(x)=\cos \left(\frac{2 k-1}{2} \pi x\right)
$$

we obtain - using Eq.(3.9) - the following relationships

$$
\begin{aligned}
& A_{m i}=\left\{\begin{array}{lll}
0 & \text { for } & i \neq m \\
1 & i=m
\end{array}\right\}, \quad B_{m i}=\left\{\begin{array}{ccc}
0 & & i \neq m \\
-\frac{\zeta}{2} & \text { for } & i=m
\end{array}\right\}, \\
& D_{m i}=\left\{\begin{array}{ccr}
0 & i \neq m \\
-\frac{\zeta^{2}+3}{3} a_{m}^{2}+\frac{\zeta}{2} & \text { for } & i=m
\end{array}\right\}, \\
& F_{m}=(-1)^{m+1} \frac{2}{a_{m}}\left[\left(1-\frac{2}{a_{m}^{2}}\right) \zeta^{2}+1\right]
\end{aligned}
$$

where

$$
a_{m}=\frac{2 m-1}{2} \pi
$$

Inserting relationships (3.13) into formulae (3.8) we may determine the coefficients $C_{m}$

$$
C_{m}\left(1-\frac{\zeta^{2}+3}{\zeta^{2}+\lambda^{2}} \frac{a_{m}^{2}}{3} G_{\vartheta}\right)=\left(1+\frac{2 I \zeta^{2}}{\zeta^{2}+\lambda^{2}} G_{I}\right)(-1)^{m+1} \frac{2}{a_{m}}\left[\left(1-\frac{2}{a_{m}^{2}}\right) \zeta^{2}+1\right]
$$

and then 


$$
C_{m}=\frac{\left(1+\frac{2 I \zeta^{2}}{\zeta^{2}+\lambda^{2}} G_{I}\right)(-1)^{m+1} \frac{2}{a_{m}}\left[\left(1-\frac{2}{a_{m}^{2}}\right) \zeta^{2}+1\right]}{1-\frac{\zeta^{2}+3}{\zeta^{2}+\lambda^{2}} \frac{a_{m}^{2}}{3} G_{\vartheta}} .
$$

The pressure distribution in the bearing clearance can be determined by applying Eqs (2.14), (3.1) and (3.16).

\section{Mechanical parameters of a multilobe conical bearing}

To determine the mechanical parameters of the bearing, let us consider the conical tapered roller bearing shown in Fig.1. The geometrical parameters of this bearing are defined by the following relationships

$$
H=1-\alpha \vartheta, \quad \alpha=\frac{M-1}{M \psi}, \quad \psi=\frac{2 \pi}{N} .
$$

The solution to relationships (3.4), (3.5), (3.10) and (3.11) can be presented as follows

$$
\begin{aligned}
& H^{*}=\frac{M-1+12 \beta^{2} \frac{M^{3}-1}{3}}{\frac{M^{2}-1}{2}+12 \beta^{2} \frac{M^{4}-1}{4}} ; \\
& P_{\infty}(\vartheta)=\frac{1}{\alpha}\left[\left(\frac{1-H}{H}+12 \beta^{2} \frac{1-H^{3}}{3 H^{3}}\right)+H^{*}\left(\frac{1-H^{2}}{2 H^{2}}+12 \beta^{2} \frac{1-H^{4}}{4 H^{4}}\right)\right], \\
& G_{\vartheta}=\frac{G_{L}^{(\vartheta)}}{G_{M}^{(\vartheta)}}, \\
& G_{I}=\frac{G_{L}^{(I)}}{G_{M}^{(I)}} .
\end{aligned}
$$

The formulae that determine the coefficients $G_{L}^{(\vartheta)}, G_{M}^{(\vartheta)}, G_{L}^{(I)}$ and $G_{M}^{(I)}$ are given in the Appendix.

Figure 2 shows the plots of the lubricant film thickness $H^{*}$ versus the coefficient of the lobe taper $M$ for different values of couple stress $l^{*}$.

From the plots depicted in Fig. 2 it can be concluded that the increase of the couple stress $\left(l^{*}>0\right)$ generates the decrease of the parameter $H^{*}$ compared to the Newtonian fluid $\left(l^{*}=0\right)$. We can observe that the value of the parameter $H^{*}$ decreases with an increasing value of the coefficient of the lobe taper $M$. 


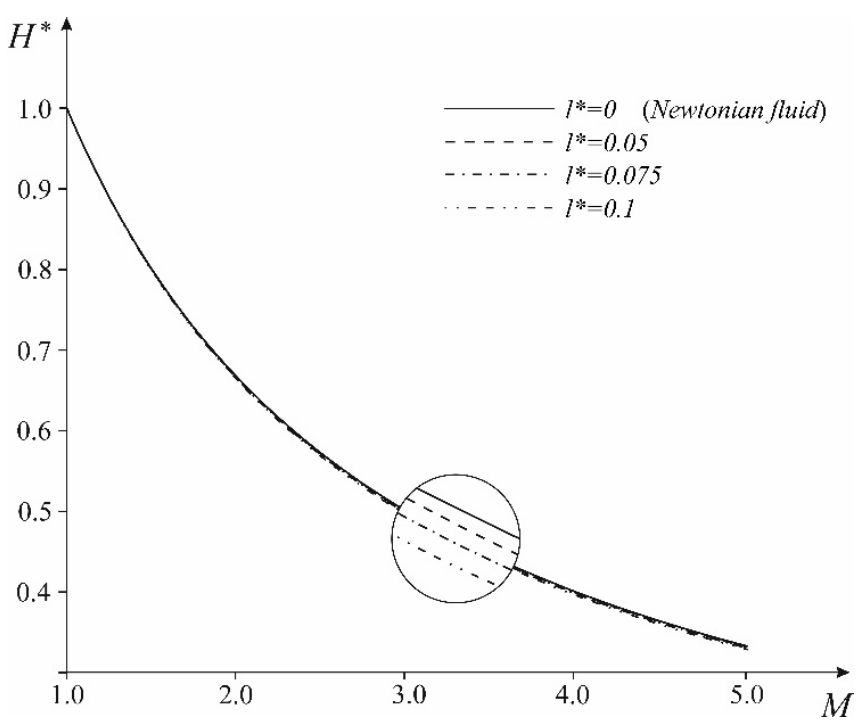

Fig.2. Dimensionless bearing thickness $H^{*}$ versus $M$ for different values of couple stress $l^{*}=0 ; 0.05 ; 0.075 ; 0.1$.

Figure 3 shows the pressure distribution $P_{\infty}(\vartheta)$ for the two-lobe bearing $(N=2)$ and for the coefficient of the lobe taper $M=2 ; 3 ; 4 ; 5$ and different values of couple stress $l^{*}=0 ; 0.05 ; 0.075 ; 0.1$.

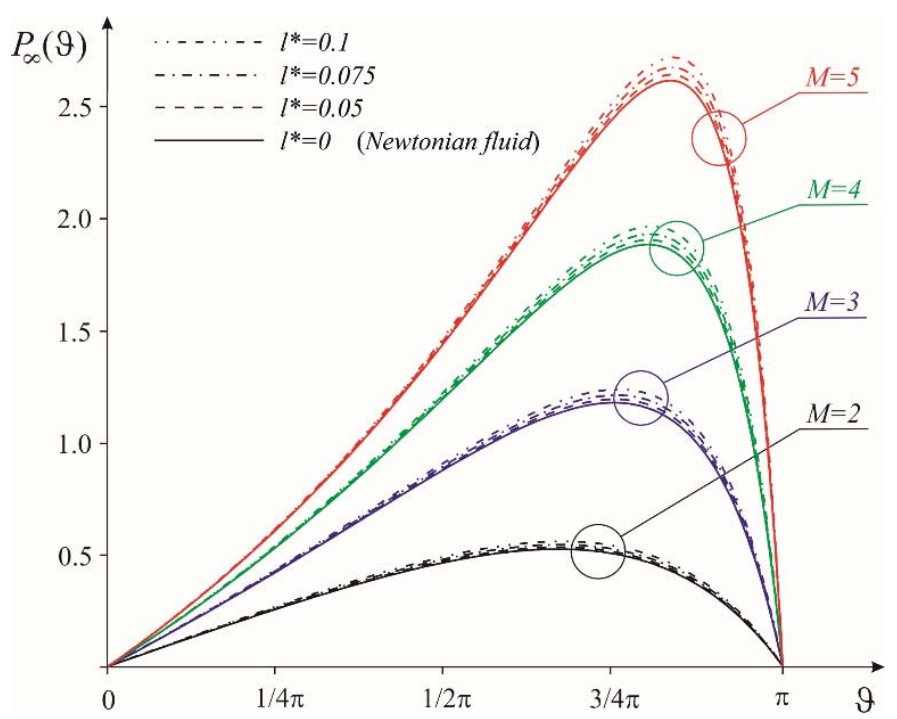

Fig.3. Dimensionless pressure $P_{\infty}(\vartheta)$ for the two-lobe bearing and for $M=2 ; 3 ; 4 ; 5$ and for different values of couple stress $l^{*}=0 ; 0.05 ; 0.075 ; 0.1$.

From Fig.3 it can be concluded that the values of the pressure $P_{\infty}(\vartheta)$ increase with the increases of the couple stress $\left(l^{*}>0\right)$ compared to the Newtonian fluid $\left(l^{*}=0\right)$. The values of the pressure $P_{\infty}(\vartheta)$ increase also with the increase of the parameter $M$. 


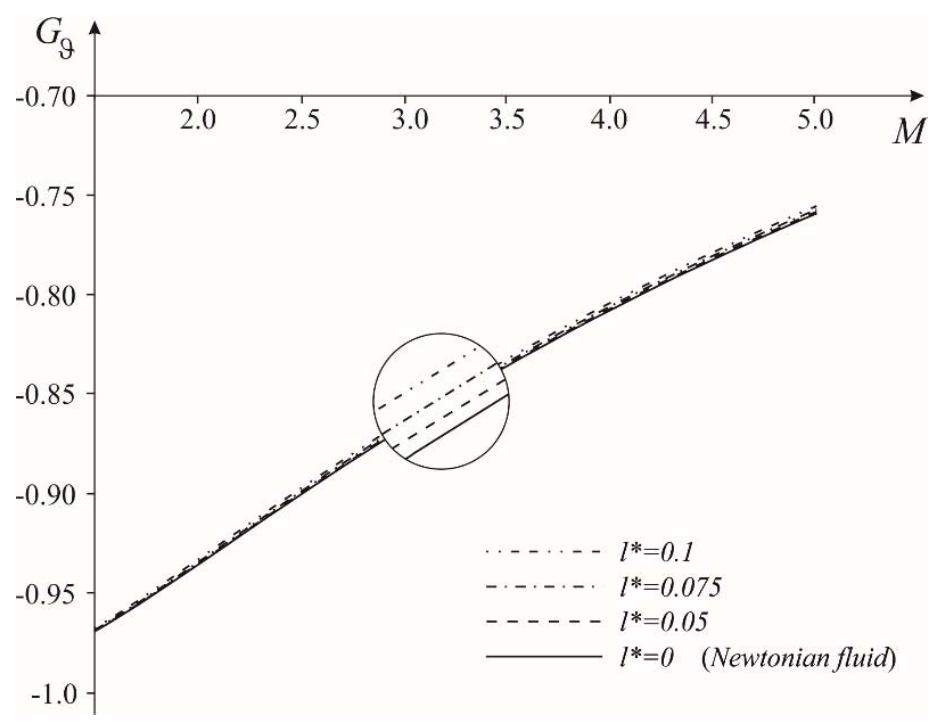

Fig.4. Plots of the parameter $G_{\vartheta}$ versus $M$ for $l^{*}=0 ; 0.05 ; 0.075 ; 0.1$ where $1<M \leq 5$.

Figures 4 and 5 show the plots of the parameters $G_{\vartheta}$ and $G_{I}$, respectively, versus the coefficient of the lobe taper $(1<M<5)$ for different values of the parameter $l^{*}$.

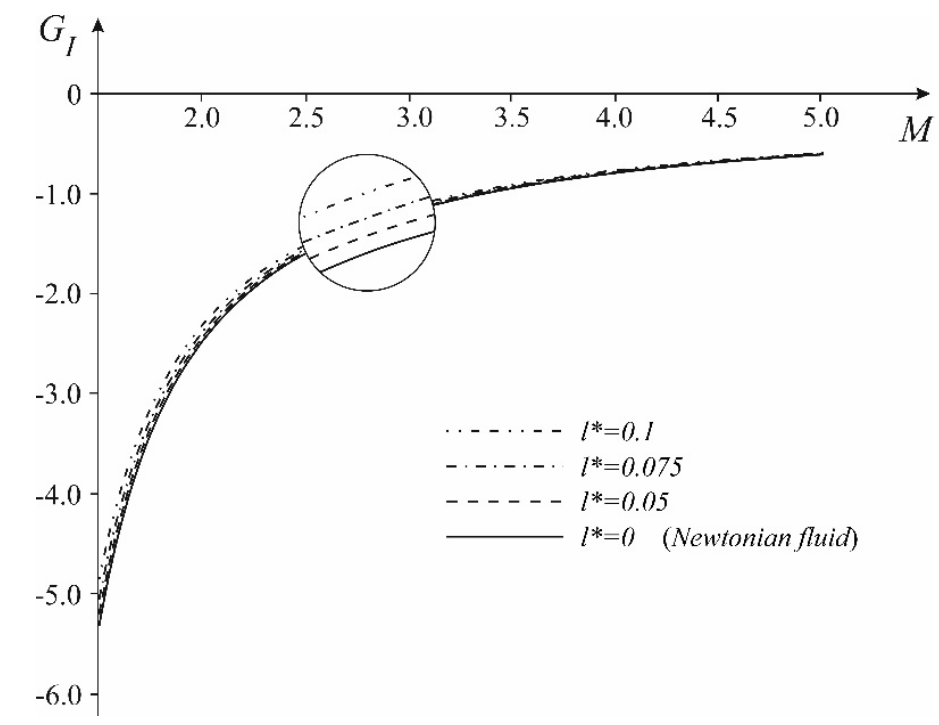

Fig.5. Plots of the parameter $G_{I}$ versus $M$ for $l^{*}=0 ; 0.05 ; 0.075 ; 0.1$ where $1<M \leq 5$.

From Figs 4 and 5 it can be concluded that the values of the coefficients $G_{\vartheta}$ and $G_{I}$ increase with the increase of the parameter $M$. The values of the coefficients $G_{\vartheta}$ and $G_{I}$ also increase with the increase of the couple stress $l^{*}$ compared to the Newtonian fluid $l^{*}=0$.

Having the values of the coefficients $G_{\vartheta}$ and $G_{I}$ one may find the formulae for the mechanical parameters of the bearing from the following relationships [3]:

- the bearing axial load 


$$
W_{A}=P_{A} W_{A \infty} K_{A},
$$

here

$$
P_{A}=\Lambda_{0} R_{a}^{2} \zeta, \quad W_{A \infty}=N \int_{0}^{\psi} P_{\infty}(\vartheta) d \vartheta, \quad K_{A}=\sum_{m=1}^{n} K_{m}
$$

and

$$
K_{m}=\frac{\frac{4}{a_{m}^{2}}\left(1+\frac{2 I \zeta^{2}}{\zeta^{2}+\lambda^{2}} G_{I}\right)\left[\left(1-\frac{2}{a_{m}^{2}}\right) \zeta^{2}+1\right]}{1-\frac{\zeta^{2}+3}{\zeta^{2}+\lambda^{2}} \frac{a_{m}^{2}}{3} G_{\vartheta}} ;
$$

- the friction torque

$$
T_{s}=T_{s 1} I_{1}+T_{s 2}\left(I_{1}-I_{2}\right) K_{A},
$$

where

$$
\begin{aligned}
& T_{s 1}=\frac{\mu \omega R_{o}^{4}}{4 h_{M} \sin \alpha}\left(1-\varepsilon^{4}\right), \quad T_{s 2}=\frac{3 \mu \omega R_{o}^{4}}{16 h_{M} \sin \alpha}(1-\varepsilon)(1+\varepsilon)^{3}, \\
& I_{1}=N \int_{0}^{\psi} \frac{1}{H} d \vartheta, \quad I_{2}=N H^{*} \int_{0}^{\psi} \frac{1}{H^{2}} d \vartheta .
\end{aligned}
$$

The values of both the magnitudes depend on the number of the bearing lobes.

\section{Conclusions}

In this paper, the inertia effects of the couple stress lubrication flowing in the multilobe conical bearing were analysed. After the analyses, the value of the parameter $H^{*}$ was calculated. The parameter $H^{*}$ is the lubricant film thickness when the pressure $P_{\infty}(\vartheta)$ attains its maximum. The graphical distribution of parameter $H^{*}$ shows that its values decrease with the increase of the parameter $M$. The increase of couple stress reduces also the value of the parameter $H^{*}$ (compared to the Newtonian fluid $l^{*}=0$ ).

The graphical interpretation of the pressure distribution $P_{\infty}(\vartheta)$ shows that its values increase with the increase of the parameter $M$ being the coefficient of the lobe taper. The values of the pressure increase also with the increase of the parameter $l^{*}$. The values of the parameters $G_{\vartheta}$ and $G_{I}$ increase with the increase of the values of the parameter $M$, the values of these coefficients increase also with the increase of the couple stress $l^{*}$ compared to the Newtonian fluid $l^{*}=0$. 


\section{Appendix}

The formula for $G_{L}^{(\vartheta)}$ has the form

$$
\begin{aligned}
& G_{L}^{(9)}=\frac{1}{\alpha^{3}}\left\{\left(\frac{1}{12}-\frac{1}{4 M^{4}}+\frac{2}{3 M^{3}}-\frac{1}{2 M^{2}}+\frac{28 \beta^{2}}{3}-\frac{2 \beta^{2}}{M^{4}}+\frac{8 \beta^{2}}{3 M^{3}}+\right.\right. \\
& \left.+\frac{6 \beta^{2}}{M^{2}}-\frac{16 \beta^{2}}{M}+4 \beta^{2} \ln \frac{1}{M}\right)+H^{*}\left(-\frac{5}{12}+\frac{1}{4 M^{4}}-\frac{1}{3 M^{3}}-\frac{1}{2 M^{2}}+\frac{1}{M}+\right. \\
& \left.-\frac{5 \beta^{2}}{2}+\frac{5 \beta^{2}}{2 M^{4}}-\frac{2 \beta^{2}}{M^{3}}-\frac{8 \beta^{2}}{M^{2}}+\frac{8 \beta^{2}}{M}+2 M \beta^{2}+6 \beta^{2} \ln \frac{1}{M}\right)+ \\
& \left.+H^{* 2}\left(-\frac{3}{16}-\frac{1}{16 M^{4}}+\frac{1}{4 M^{2}}-\frac{9 \beta^{2}}{4}-\frac{3 \beta^{2}}{4 M^{4}}+\frac{3 \beta^{2}}{M^{2}}-\frac{1}{4} \ln \frac{1}{M}-3 \beta^{2} \ln \frac{1}{M}\right)\right\} .
\end{aligned}
$$

The formula for $G_{L}^{(I)}$ has the form

$$
\begin{aligned}
& G_{L}^{(I)}=-\frac{1}{\alpha^{2}}\left(-\frac{M^{4}+1}{4 M^{4}}+\frac{11 \beta^{2} M^{3}+1}{3 M^{3}}+\frac{\beta^{2}\left(20 M^{2}-1\right)}{3 M^{4}}+\frac{80 \beta^{4}-28 \beta^{2} M-80 M^{3} \beta^{4}}{3 M^{2}}+\right. \\
& +\frac{22 \beta^{3}}{\sqrt{3}}\left(\operatorname{arctanh} \frac{1}{2 \sqrt{3} M \beta}-\operatorname{arctanh} \frac{1}{2 \sqrt{3} \beta}\right)+H^{*}\left\{\frac{M^{4}+1}{8 M^{4}}+\frac{31 \beta^{2}}{12}+\frac{3 \beta^{2}-M^{2}}{4 M^{4}}+\right. \\
& \left.\left.-\frac{10 \beta^{2}}{3 M^{2}}-\frac{20 \beta^{4}}{M^{2}}+20 M^{2} \beta^{4}+\frac{11}{6} \beta^{2}\left[\ln \left(\frac{1}{M^{2}}-12 \beta^{2}\right)-\ln \left(1-12 \beta^{2}\right)\right]\right)\right\} .
\end{aligned}
$$

The formula for $G_{M}^{(\vartheta)}=G_{M}^{(I)}$ has the form

$$
\begin{aligned}
& G_{M}^{(\vartheta)}=G_{M}^{(I)}=\frac{1}{2 \alpha M}\left[2\left(M-1-4 \beta^{2}+6 M \beta^{2}-2 M^{3} \beta^{2}\right)+\right. \\
& \left.+H^{*}(M-1)^{2}\left(1+2 M\left(M^{2}+2 M+3\right)\right)+2 M \ln \frac{1}{M}\right] .
\end{aligned}
$$

\section{Nomenclature}

$f(l, h)$ - function given by formula (2.9)

$f\left(l^{*}, h\right)$ - function given by formula $(2.12)_{2}$

$g(l, h)$ - function given by formula $(2.9)_{1}$

$g\left(l^{*}, h\right)$ - function given by formula $(2.12)_{2}$

$h$ - gap thickness

$h_{m}$ - minimal gap thickness 
$h_{M}-$ maximal gap thickness

$M$ - coefficient of the lobe taper

$N$ - number of the bearing lobes

$P_{A} \quad$ - parameter of the axial load carrying capacity

$p$ - pressure

$R$ - radius of the shaft

$R_{a}$ - averaged radius of the shaft

$W_{A}$ - axial load carrying capacity of the bearing

$W_{A \infty}$ - dimensionless axial load without annular flow

$x, \vartheta, y$ - orthogonal curvilinear coordinate system

$\varepsilon \quad$ - bearing taper

$\zeta$ - relative radial length of the bearing

$\lambda$ - relative axial length of the bearing

$\psi \quad-$ arc span of a lobe of the bearing

$\omega$ - angular velocity

\section{References}

[1] Walicka A. (2002a): Rheodynamics of Non-Newtonian Fluids Flow in Straight and Curved Channels (in Polish). - Zielona Góra: University Press.

[2] Walicka A. (2002b): Rotational Flows of Rheological Complex Media in Narrow Annular Channels (in Russian). - Zielona Góra: University Press.

[3] Walicki E. (2005): Rheodynamics of Slide Bearings Lubrication (in Polish). - Zielona Góra: University Press.

[4] Walicka A. (2017): Rheology of Fluid in Mechanical Engineering. - Zielona Góra: TU Press.

[5] Walicka A. (1994): Micropolar Flow in a Slot Between Rotating Surfaces of Revolution. - Zielona Góra: TU Press.

[6] Lipscomb C.C. and Denn M.M. (1984): Flow of Bingham fluids in complex geometries. - J. Non-Newt. Fluid Mech., vol.14, No.3, pp.337-349.

[7] Dorier C. and Tichy J. (1992): Behaviour of a Bingham-like viscous fluid in lubrication flows. - J. Non-Newt. Fluid Mech., vol.45, No.3, pp.291-350.

[8] Wada S. and Hayashi H. (1971a): Hydrodynamic lubrication of journal bearings by pseudo-plastic lubricants, (Pt 1, Theoretical studies). - Bull. JSME, vol.14, No.69, pp.268-278.

[9] Wada S. and Hayashi H. (1971b): Hydrodynamic lubrication of journal bearings by pseudo-plastic lubricants. (Pt 2, Experimental studies). - Bull. JSME, vol.14, No.69, pp.279-286.

[10] Swamy S.T.N., Prabhu B.S. and Rao B.V.A. (1975): Stiffness and damping characteristics of finite width journal bearing with a non-Newtonian film and their application to instability prediction. - Wear, vol.32, pp.379-390.

[11] Rajalingham C., Rao B.V.A. and Prabu S. (1978): The effect of a non-Newtonian lubricant on piston ring lubrication. - Wear, vol.50, pp.47-57.

[12] Stokes V.K. (1966): Couple-stresses in fluids. - Phys. Fluids, vol.9, No.9, pp.1709-1715.

[13] Basavaraja J.S., Sharma S.C. and Jain S.C. (2008): Performance of an orifice compensated two-lobe hole-entry hybrid journal bearing. - Advances in Tribology, vol. 2008, Article ID 871952, 10 pages.

[14] Kushare P.B and Sharma S.C, (2014): Nonlinear transient stability study of two lobe symmetric hole entry worn hybrid journal bearing operating with non-Newtonian lubricant. - Tribology International, vol.69, pp.84-101.

[15] Sharma S.C. and Rajput A.K. (2012): Influence of micropolar lubrication on the performance of 4-pocket capillary compensated conical hybrid journal bearing. - Advances in Tribology, vol.2012, Article ID 898252, 18 pages, doi:10.1155/2012/898252. 
[16] Rana N.K., Gautam S.S. and Verma S. (2014): Static characteristics of conical hydrostatic journal bearing under micropolar lubrication. - J. Inst. Eng. India Ser. C vol.95, No.4, pp.375-381.

[17] Walicki E. and Walicka A. (1994): Analysis of an incompressible Newtonian flow in a slot of the curvilinear thrust bearing. - Mechanika, ZN WSI Zielona Góra, No.20, pp.33-60.

[18] Walicki E., Walicka A., Jurczak P. and Wojnarowski J. (2015): Mechanical parameters for a multilobe conical bearing lubricated with a couple-stress fluid. - Machine Dynamics Research, vol.39, No.4, pp.43-54.

Received: June 17, 2018

Revised: September 29, 2018 